\title{
X-ray study of krypton and xenon under pressure reveals the mechanism of martensitic transformations
}

\author{
Angelika Dorothea Rosa ${ }^{1}$, Agnes Dewaele ${ }^{2}$, Gaston Garbarino ${ }^{1}$, Irifune Tetsuo ${ }^{3}$, Olivier Mathon ${ }^{1}$, \\ Mohamed Ali Bouhifd ${ }^{4}$ \\ ${ }^{1}$ ESRF, Grenoble, France; \\ ${ }^{2}$ CEA, Arpajon Cedex, France; \\ ${ }^{3}$ Geodynamics Research Center, Ehime University, Matsuyama, Japan; \\ ${ }^{4}$ 9. Laboratoire Magmas et Volcans, Université Clermont Auvergne, Clermont-Ferrand, France; \\ arosa@esrf.fr
}

The martensitic transformation is a fundamental physical phenomenon at the origin of important industrial applications. However, the underlying microscopic mechanism, which is of critical importance to explain the outstanding mechanical properties of martensitic materials, is still not fully understood. This is because for most martensitic materials the transformation is a fast process that makes in situ studies extremely challenging. Noble solids krypton and xenon undergo a progressive pressure induced fcc to hcp martensitic transition with a very wide coexistence domain. Here, we took advantage of this unique feature to study the detailed mechanism of the transformation by employing in situ X-ray diffraction and absorption. We evidenced a four stages mechanism where the lattice mismatch between the fcc and hcp forms plays a key role in the generation of strain. We also determined precisely the effect of the transformation on the compression behavior of these materials.

Keywords: martensitic-transition, krypton, xenon, high-pressure, XAS/XRD 